\title{
The Possibility of Automatic Intercalibration for Russian Satellites by Histogram Analysis
}

\author{
Alexandr V. Kashnitskiy ${ }^{1}$, Evgeny A. Loupian ${ }^{1}$, Alexey A. Mazurov ${ }^{1}$, Alexey M. Matveev ${ }^{1}$ \\ ${ }^{1}$ Space Research Institute, Russian Academy of Sciences, Moscow, Russia \\ alexei@d902.iki.rssi.ru
}

\begin{abstract}
Satellite instruments calibration is critical for detection and quantification of changes in the Earth's environment, and monitoring climate and land cover change. To meet the stringent needs of such applications, onboard instruments must provide reliable, accurate, and consistent measurements over time. This work is about one multisatellite approach for data calibration. Method is based on histogram comparing of series of two simultaneous pairs satellite images, where one is reference and other evaluated.
\end{abstract}

Keywords: remote sensing, satellite data, in-orbit radiometric calibration, cross-calibration 


\title{
АНАЛИЗ ВОЗМОЖНОСТИ АВТОМАТИЧЕСКОЙ КРОСС-КАЛИБРОВКИ ДАННЫХ РОССИЙСКИХ СПУТНИКОВ МЕТОДОМ АНАЛИЗА ГИСТОГРАММ
}

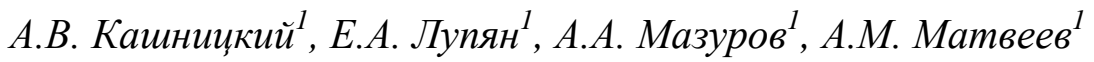 \\ ${ }^{1}$ Институт космических исследований РАН, Москва, Россия \\ alexei@d902.iki.rssi.ru
}

\begin{abstract}
Аннотация
Калибровка спутниковых приборов имеет решающее значение для обнаружения и количественной оценки изменений в окружающей среде Земли, а также для мониторинга изменения климата и земного покрова. Для удовлетворения строгих требований таких задач бортовые приборы должны обеспечивать надежные, и точные последовательные измерения. В работе предложен подход для кросскалибровки спутниковых данных. Метод основан на сравнении гистограмм серий одновременных пар спутниковых изображений, где одно является эталонным, а другое оценивается.
\end{abstract}

Ключевые слова: ДЗ3, кросскалибровка, полетная калибровка, анализ гистограмм

\section{Введение}

При работе со спутниковыми данными в настоящее время интересует их радиометрические параметры как измерительных инструментов для использования в различного рода задачах мониторинга изменений характеристик поверхности. Это оценки различных вегетационных индексов, цветность поверхности, температура или влажность почвы, интенсивность пожаров и др. В этом вопросе наиболее существенную роль играют калибровочные характеристики приборов, их изменение во время функционирования космических аппаратов ДЗ3 и их периодическое уточнение. Этой проблеме посвящено большое количество исследований и публикаций, поскольку наличие специальных возможностей для проведения качественной калибровки непосредственно на борту КА $[1,2,3,4,5,6]$ сильно усложняет компоновку аппаратуры, а также значительно удорожает производственные и эксплуатационные затраты. В современных системах ДЗЗ в целях непрерывного детального мониторинга земной поверхности запускаются массово малогабаритные КА для ежедневного мониторинга поверхности (например, система малых спутников Glove Planet Labs). Часть приборов на российских и зарубежных КА также не имеет калибровочной схемы на борту [2].

\section{Метод калибровки сравнительным анализом гистограмм}

В данной работе предлагается метод оценки измерительных характеристик (калибровочной кривой) прибора путем сравнения гистограмм изображений одних и тех же районов на поверхности Земли с различных аппаратов. В отличие от других авторов [3], предлагается использовать калиброванные, но не скорректированные на атмосферное влияние, 
данные. Суть предлагаемого метода сводится к приведению гистограммы яркостей изображения, снятого одним прибором к гистограмме изображения того же точно объекта (территории), полученного другим прибором (эталоном). Таблица преобразования яркости одного изображения в яркость на другом изображении (look-up-table или LUT) строится путем сопоставления частичных интегралов площадей гистограмм изображений, приведенных к одинаковому пространственному разрешению. Если сравниваемые приборы хорошо откалиброваны, изображения нормализованы в одинаковый диапазон яркостей (для каждого прибора), то такие таблицы LUT будут одинаковые для всех исследуемых пар одновременных снимков. В частности, если калибровки данных линейные (что обычно принято для хранящихся в архивах данных), то и построенные LUT будут линейными функциями.

Для проведения такого анализа очевидными существенными факторами [4], влияющими на точность сопоставления, являются: совпадение геометрии и времени съемки, идентичность спектральной функции чувствительности канала съемочной аппаратуры. В таком случае сможем получить соответствие значений яркости исследуемого изображения калиброванным значениям эталона. В реальности этим идеальным условиям практически невозможно удовлетворить. Однако некие «псевдоиделаьные» условия можно рассмотреть, а именно:

- выберем в качестве эталонов изображения, принятые геостационарным спутником Meteosat 11 с периодичностью съемки 30 минут.

- на изображении исследуемых аппаратов выбираем участки с углом зрения, близким к углу зрения геостационара.

- рассматривать будем каналы с близкой частотной характеристикой [5]..

В работе используются следующие данные в периоде сентябрь 2018г - февраль 2019г.:

- геостационар Метеосат 11, прибор SIVIRI, канал в видимом спектре 0.6 мкм. Разрешение 3 км. -спутник TERRA прибор MODIS, канал 1 в видимом спектре. Разрешение 250м.

-спутник «Метеор-2м», прибор КМСС, камера МСУ-100, №2, канал 2. Разрешение 60м.

Данные прибора MODIS являются хорошо калиброванными и в данной работе представляют интерес для выявления требований к условиям сопоставления данных при проведении кросскалибровки.

Все данные и результаты получены из архивов ЦКП «ИКИ-Мониторинг» с использованием сервисов доступа к данным и их обработки BЕГА-Science и единой системы работы со спутниковыми данными ФГБУ "НИЦ "Планета" [7,8].

Рассмотрим построение ряда LUT по эталонным и исследуемым изображениям для последующего формирования калибровочной функции исследуемого прибора (если такое возможно). Для исследования выберем регион вблизи экваториальной точки стояния спутника Метеосат 11 с описывающим прямоугольником (12 гр.в.д., +30 гр в.д.), (3 гр. ю.ш., 15 гр.ю.ш.). Регион выбран так из условия наличия данных в количестве более 10 сцен в указанном Центре.

При сопоставлении гистограмм данные более высокого разрешения MODIS, KMCC приводятся усреднением к соответствующему разрешению данных Метеосат. Для построения гистограмм использовались участки сцен прибора КМСС 100 вправо по направлению полета от надира (в восточном направлении) до углов съемки 65-75 градусов. Для данных MODIS - такие же условия, с разными углами съемки. Контуры регионов на снимках выбирались так, что углы визирование MODIS и KMCC не отличались во всех точках снимка более чем на 5 градусов от угла визирования с Meteosat.

Естественно ожидать в таких условиях проявления неоднородности геометрических условий съемки (не выполняется условие одинакового угла наблюдения по всему изображению с разных аппаратов в момент съемки) на сопоставляемые результаты и «схожесть» вычисляемых LUT, тем не менее, для получения статистически достаточно представительной гистограммы, пришлось этим условием в данном исследовании пожертвовать. Также время съемки отличается на интервал до 15 минут. Ниже будет показано влияние этих факторов на результаты кросскалибровки. 


\section{Применение анализа гистограмм для приборов MODIS по отношению к SEVIRI}

Для начала рассмотрим взаимное сопоставление гистограмм для одновременной съемки одной и той же территории под разными углами наблюдения. На рис.1 красной линией обозначена LUT сопоставления гистограмм для 2-х изображений территории на Экваторе посередине между точками стояния геостационаров Meteosat 8 и 11 в момент когда Солнце для этой территории в зените, А синей линией нарисована LUT для случая съмки территории в надир спутником Метеосат 11 и она же наклонно спутником Метеосат 8 (Солнце также в Зените). Видно, что в первом случае преобразование практически линейное, несмотря на съемку сцены с противоположных сторон под одинаковыми углами, а во втором случае съемки в надир и под углом LUT заметно искривляется. На этом же рисунке салатовой и зеленой линиями приведены LUT для снимков, сделанных в надир с Метеосат 11 и отстоящих по времени друг от друга в 30 и 60 минут. Мы видим как влияет временная удаленность снимков на искривление LUT. Черным пунктиром изображена LUT самоподобного преобразования гистограммы изображения.

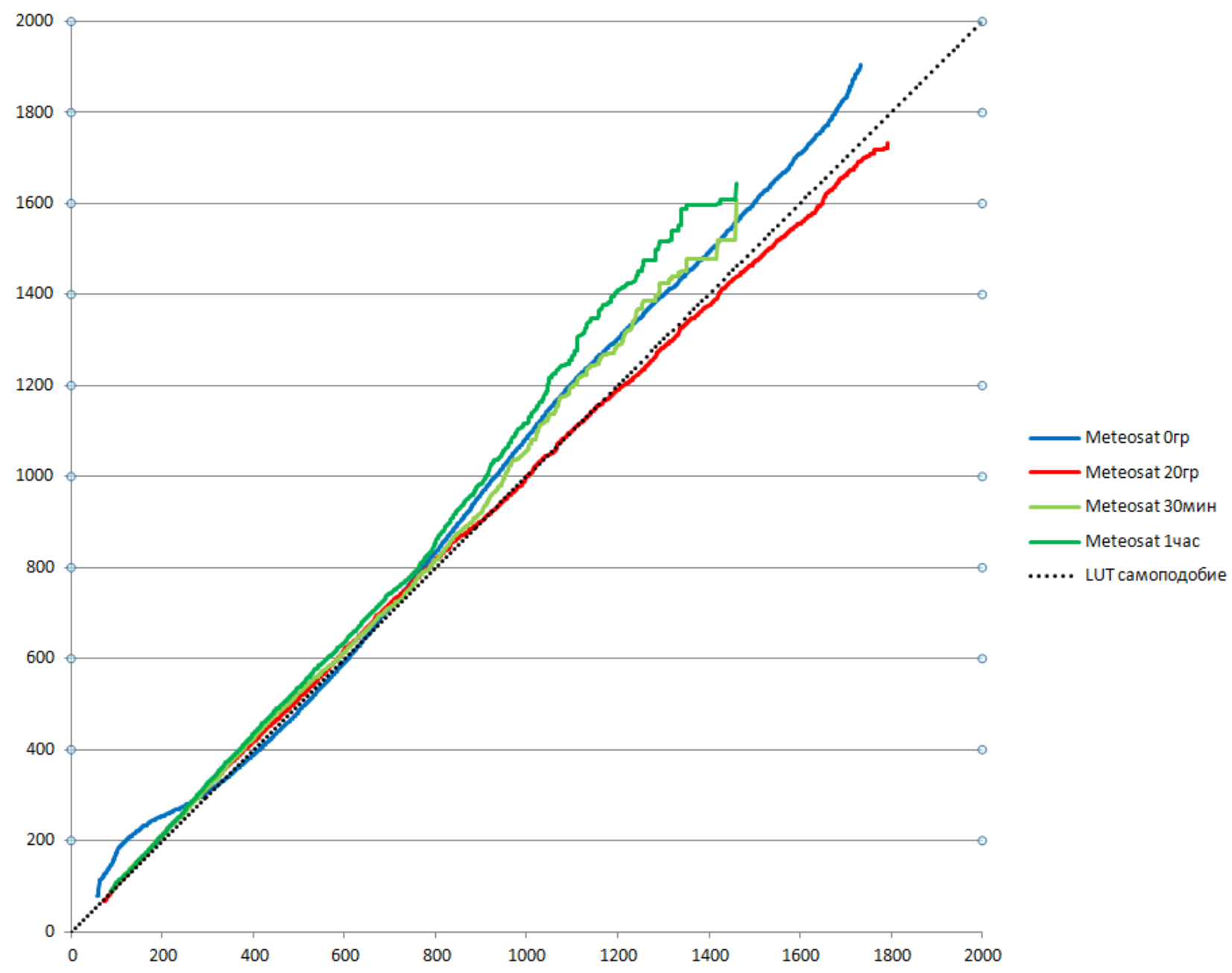

Рис. 1. LUT сопоставления гисторамм Meteosat 8 - Meteosat 11

Для того чтобы смоделировать, как должна вести себя LUT прибора с неизвестной калибровкой в условиях одновременной с геостационаром съемки территории, рассмотрим построение таких LUT для ряда изображений с калиброванными данными MODIS. Ha рис. 2 разными цветами представлены графики следующих наборов LUT:

- синим цветом обозначены съемки с одинаковым углом зрения и временным интервалом около 15 мин.

- красным цветом обозначены съемки с одинаковым углом зрения и временным интервалом менее 5 мин. 
- зеленым цветом обозначены съемки с разным углом зрения (MODIS снимает в западном направлении) и временным интервалом менее 5 мин.

Хорошо видно, что при близкой по времени и углу съемке преобразование сопоставляемых гистограмм имеет практически одинаковый линейный вид для всего временного ряда (ошибка от среднего не превышает 0.03 в единицах коэффициента спектральной яркости).

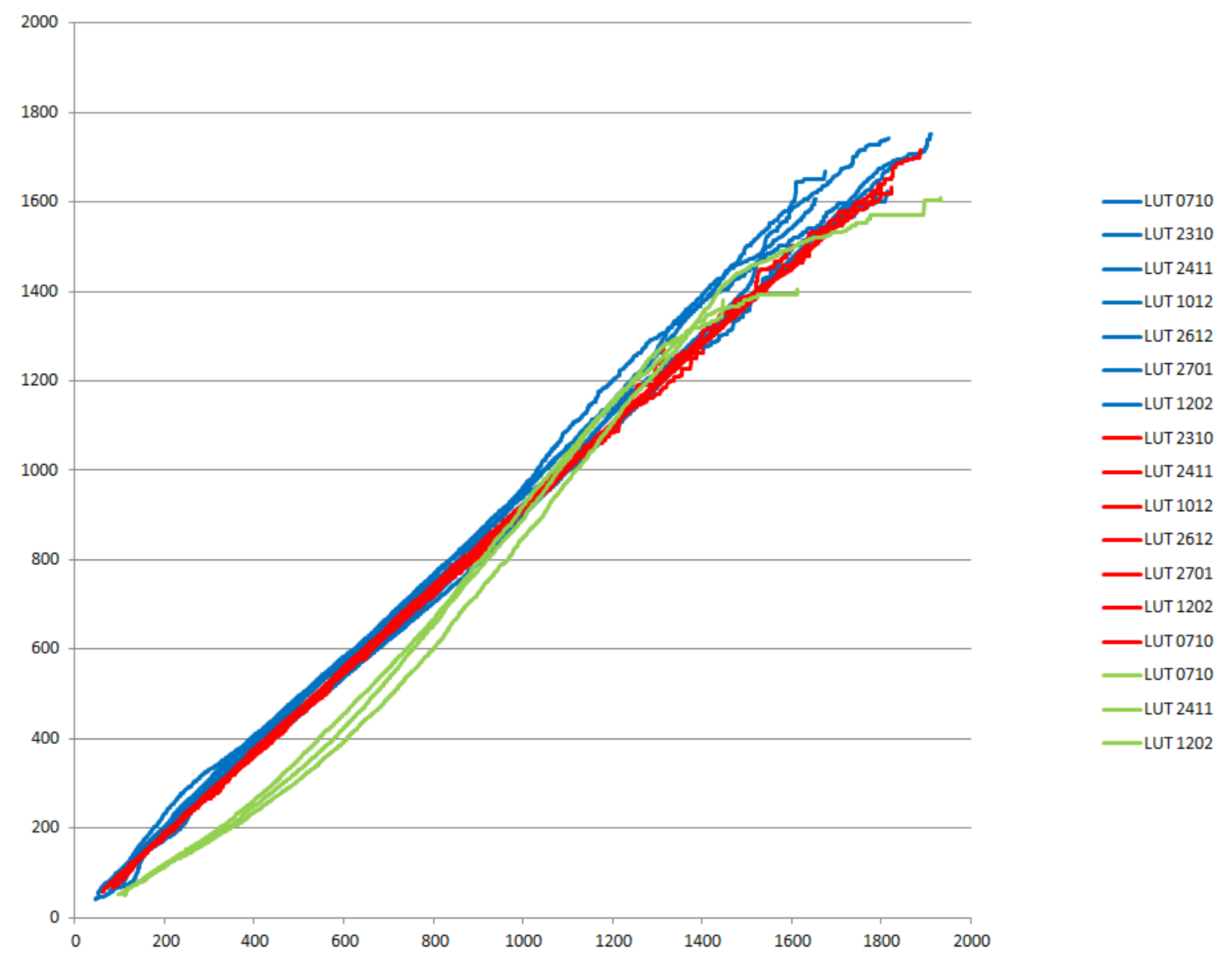

Рис. 2. LUT сопоставления гисторамм MODIS - Meteosat 11. Цифрами обозначены даты съемок с октября 2018 г. (0710) по февраль 2019 г. (1202) 


\section{Применение анализа гистограмм для прибора КMCC по отношению к SEVIRI}

Теперь рассмотрим группу линий на Рис. 3, представляющих аналогичные Рис. 2 таблицы LUT сопоставления гистограмм изображений приборов КМСС и SIVIRI.

Синим цветом нарисованы графики для случая временного интервала между снимками с разных аппаратов более 10 минут, а красными - менее 3 минут. Видно, что имея тенденцию к одинаковому наклону, линии отстоят друг от друга на большее расстояние, чем в случае с данными MODIS. При этом временного тренда ухода линий в каком либо направлении нет.

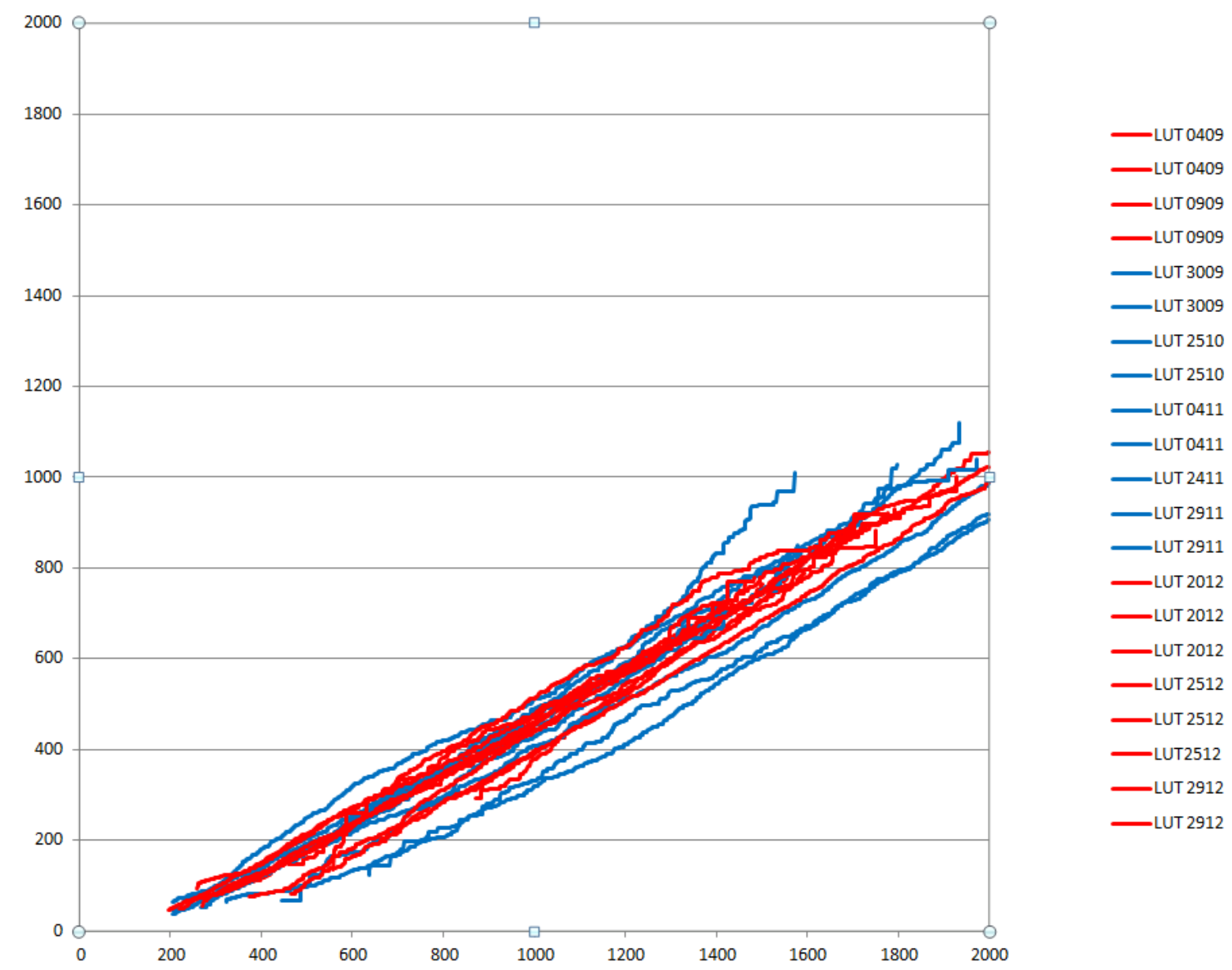

Рис. 3. LUT сопоставления гисторамм КМСC - Meteosat 11. Цифрами обозначены даты съемок с сентября 2018 г. (0409) по декабрь 2019 г. (2912)

\section{Выводы}

В итоге, сравнивая поведение таблиц LUT для MODIS и KMCC, можно делать выводы о стабильности работы регистрирующей аппаратуры и о потенциальной возможности использовать предлагаемый подход к калибровке измерений приборов. В приведенном случае видно, что применяемые калибровки данных прибора КМСС приводят к ошибкам в измерениях, нестабильны на длительном (несколько дней) интервале времени и требуют регулярной коррекции [6]. Если использовать кросскалибровку приведенным методом, то, применяя посчитанные LUT, можно проводить краткосрочные коррекции калибровочных данных. Следует заметить, что этот метод может быть автоматизирован как для перекалибровки архивных данных, так и для планирования оперативной автоматической калибровки.

Работа выполнена при поддержке РФФИ, проект № 17-05-41152 РГО_а, с использованием ресурсов ЦКП «ИКИ-Мониторинг» [7] (поддерживается Минобрнауки в рамках темы «Мониторинг», госрегистрация № 01.20.0.2.00164). 


\section{References}

[1] Zhou G., Li C., Yue T., Jiang L., Liu N., Sun Y., Li M. An overview of in-orbit radiometric calibration oftypical satellite sensors, Remote Sensing and Spatial Information Sciences, 2015, Vol. XL-7/W4, 2015 International Workshop on Image and Data Fusion, 21 - 23 July 2015, Kona, Hawaii, USA.

[2] Chander G., Hewison T. J., Fox N., Wu X., Xiong X., William J. Overview of Intercalibration of Satellite Instruments, IEEE Transactions on geoscience and remote sensing, March 2013, 51(3), pp 1056-1080.

[3] Yang Z., Mueller R. Unbiased histogram matching quality measure for optimal radiometric normalization, ASPRS 2008 Annual Conference, Portland, Oregon April 28 - May 2, 2007.

[4] LiuJ.-J., Li Z.,. Qiao Y.-L,. Liu Y.-J,. Zhang Y.-X. A new method for cross-calibration of two satellite sensors, IEEE Int. J. Remote sensing, 10 December, 2004, 25(23), pp. 5267-5281.

[5] T.V. Kondratieva, B.S. Zhukov, I.V. Polyansky1, A.A. Forsh. Comparison of reflectances of natural objects from Meteor-M No.1 Multispectral Satellite Imaging System and Terra MODIS spectroradiometer Sovremennye Problemy Distantsionnogo Zondirovaniya Zemli iz Kosmosa, 2015, 12(1), pp. 215-224. (In Russian).

[6] Sterckx S., Adriaensen S., Dierckx W., Bouvet M. In-Orbit Radiometric Calibration and Stability Monitoring of the PROBA-V Instrument, Remote Sens. 2016, 8, 546; DOI:10.3390/rs8070546.

[7] Loupian E.A., Proshin A.A., Burtsev M.A., Balashov I.V., Bartalev S.A., Efremov V.Yu., Kashnitskiy A.V., Mazurov A.A., Matveev A.M., Sudneva O.A., Sychugov I.G., Tolpin V.A., Uvarov I.A. IKI center for collective use of satellite data archiving,processing and analysis systems aimed at solving the problems of environmental study and monitoring, Sovremennye problemy distantsionnogo zondirovaniya Zemli iz kosmosa, 2015, Vol. 12, No 5, pp. 263-284. (In Russian).

[8] Loupian E.A., Milexin O.E., Antonov V.N., Kramareva L.S., Bourtsev M.A., Balashov I.V., Tolpin V.A., Solovyev V.I. System of operation of joint information resources based on satellite data in the Planeta Research Centers for Space Hydrometeorology, Russian Meteorology and Hydrology. 2014, Vol. 39, Issue 12, pp. 847-853. DOI: $10.3103 / \mathrm{S} 1068373914120103$. 